\title{
Charged Higgs production with a $W$ boson or a top quark
}

Nikolaos Kidonakis*†

Department of Physics, Kennesaw State University, USA

E-mail: nkidonak@kennesaw.edu

I present theoretical results for charged Higgs production in association with a $W$ boson or a top quark at the LHC. I calculate higher-order threshold corrections and show that they are very significant. I present detailed results for total cross sections as well as transverse-momentum and rapidity distributions for various LHC energies.

The European Physical Society Conference on High Energy Physics 5-12 July 2017

Venice, Italy

*Speaker.

${ }^{\dagger}$ This material is based upon work supported by the National Science Foundation under Grant No. PHY 1519606. 


\section{Introduction}

A charged Higgs discovery would be an unmistakable sign of new physics. Thus, the search for charged Higgs bosons is a significant part of current collider programs. Charged Higgs bosons appear in 2-Higgs doublet models, such as the MSSM, and the LHC has good potential for discovery of such particles via a variety of production modes. I discuss two important production processes: the associated production of a charged Higgs with a top quark, via the partonic process $b g \rightarrow t H^{-}$, and the associated production of a charged Higgs with a $W$ boson, via the partonic process $b \bar{b} \rightarrow H^{-} W^{+}$.

I show that higher-order corrections are significant for both processes. Given the very massive final states, soft-gluon corrections are important. I calculate these soft-gluon corrections through NNLO. In Section 2, I present approximate NNLO (aNNLO) results for total cross sections and top-quark differential distributions in $t H^{-}$production. In Section 3, I present aNNLO results for charged-Higgs differential distributions in $H^{-} W^{+}$production.

\section{2. $t H^{-}$production}

The top quark is the heaviest known elementary particle and it has unique properties, such as decay before hadronization. The lowest-order cross section for the process $b g \rightarrow t H^{-}$is proportional to $\alpha \alpha_{s}\left(m_{b}^{2} \tan ^{2} \beta+m_{t}^{2} \cot ^{2} \beta\right)$ where $\tan \beta=v_{2} / v_{1}$ is the ratio of the vevs of the two Higgs doublets. NLO corrections for this process were calculated in Ref. [1]. It has long been known that soft-gluon corrections are important for this process [2-4] as well as for related top-quark processes [5].

For the process $b\left(p_{b}\right)+g\left(p_{g}\right) \longrightarrow t\left(p_{t}\right)+H^{-}\left(p_{H}\right)$ we define $s=\left(p_{b}+p_{g}\right)^{2}, t=\left(p_{b}-p_{t}\right)^{2}$, $u=\left(p_{g}-p_{t}\right)^{2}$ and $s_{4}=s+t+u-m_{t}^{2}-m_{H}^{2}$. At partonic threshold $s_{4} \rightarrow 0$.

Soft-gluon corrections appear as $\left[\frac{\ln ^{k}\left(s_{4} / m_{H}^{2}\right)}{s_{4}}\right]_{+}$where, for the order $\alpha_{s}^{n}$ corrections, $k \leq 2 n-$ 1. We resum these soft corrections for the double-differential cross section. At NLL accuracy this resummation was presented in [2]. At NNLL accuracy we need two-loop soft anomalous dimensions [3,6]. The resummed cross section can be expanded to NNLO using general techniques [7]; aNNLO results from NNLL resummation for this process were first presented in Ref. [3]. More recently, aNNLO total cross sections and top-quark transverse-momentum $\left(p_{T}\right)$ and rapidity distributions in $t H^{-}$production were presented in Ref. [4].

To derive soft-gluon resummation, we first take moments of the partonic cross section with moment variable $N: \hat{\sigma}(N)=\int\left(d s_{4} / s\right) e^{-N s_{4} / s} \hat{\sigma}\left(s_{4}\right)$. Then, the moment-space factorized expression for the cross section in 4- $\varepsilon$ dimensions is:

$$
\hat{\sigma}^{b g \rightarrow t H^{-}}(N, \varepsilon)=\left(\prod_{i=b, g} J_{i}(N, \mu, \varepsilon)\right) H^{b g \rightarrow t H^{-}}\left(\alpha_{s}(\mu)\right) S^{b g \rightarrow t H^{-}}\left(\frac{m_{H}}{N \mu}, \alpha_{s}(\mu)\right),
$$

where $\mu$ is the scale, $J_{i}$ are jet functions, $H^{b g \rightarrow t H^{-}}$is the hard-scattering function, and $S^{b g \rightarrow t H^{-}}$is the soft function [4].

The soft function $S^{b g \rightarrow t H^{-}}$satisfies the renormalization group equation

$$
\left(\mu \frac{\partial}{\partial \mu}+\beta\left(g_{s}, \varepsilon\right) \frac{\partial}{\partial g_{s}}\right) S^{b g \rightarrow t H^{-}}=-2 S^{b g \rightarrow t H^{-}} \Gamma_{S}^{b g \rightarrow t H^{-}} .
$$


The soft anomalous dimension $\Gamma_{S}^{b g \rightarrow t H^{-}}$is calculated at two loops [3] and it controls the evolution of $S^{b g \rightarrow t H^{-}}$, resulting in the exponentiation of logarithms of $N[3,4]$.

The aNNLO soft-gluon corrections from the resummation are:

$$
\frac{d^{2} \hat{\sigma}_{\mathrm{aNNLO}}^{(2) b g \rightarrow t H^{-}}}{d t d u}=F_{\mathrm{LO}}^{b g \rightarrow t H^{-}} \frac{\alpha_{s}^{2}}{\pi^{2}} \sum_{k=0}^{3} C_{k}^{(2)}\left[\frac{\ln ^{k}\left(s_{4} / m_{H}^{2}\right)}{s_{4}}\right]_{+},
$$

where $F_{L O}^{b g \rightarrow t H^{-}}$is the leading-order term and the $C_{k}^{(2)}$ coefficients are given in [4].
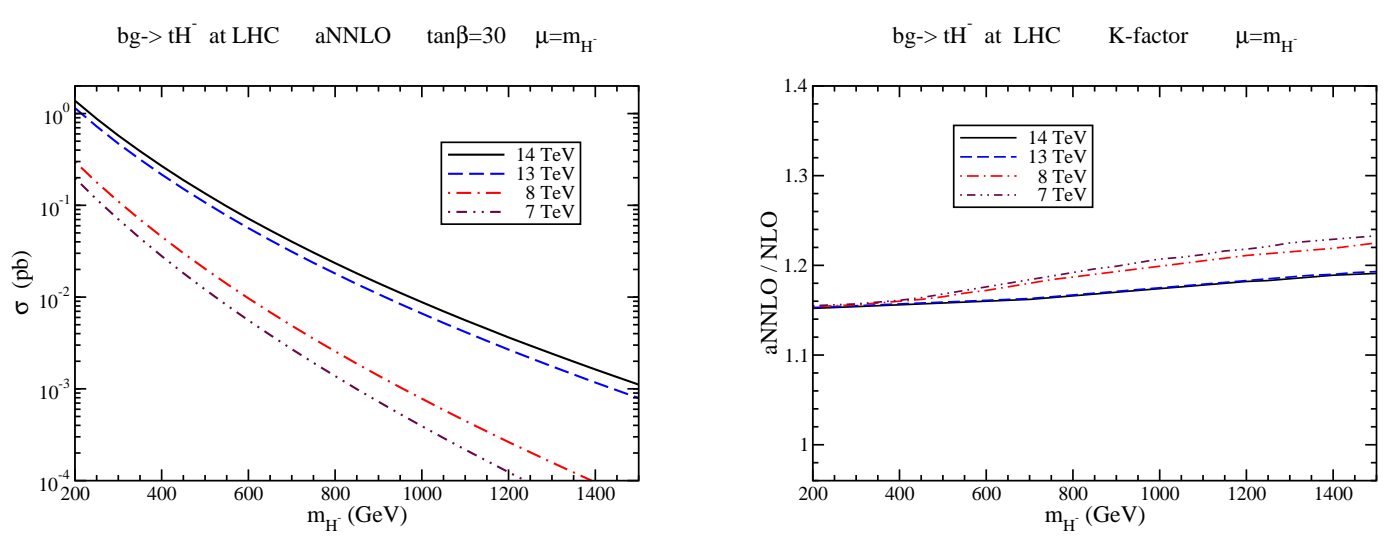

Figure 1: Total cross sections with $\tan \beta=30$ (left), and $K$ factors (right) for $t H^{-}$production.

We begin with the total cross sections for $t H^{-}$production using MMHT2014 NNLO pdf [8]. In the left plot of Fig. 1 we show the total cross sections at 7, 8, 13, and $14 \mathrm{TeV}$ LHC energies with $\tan \beta=30$ for a wide range of charged Higgs masses. The aNNLO/NLO $K$-factors are shown in the plot on the right. The aNNLO corrections are large and can reach $20 \%$ or more for high charged Higgs masses. The corrections are larger for lower LHC energies, closer to threshold.
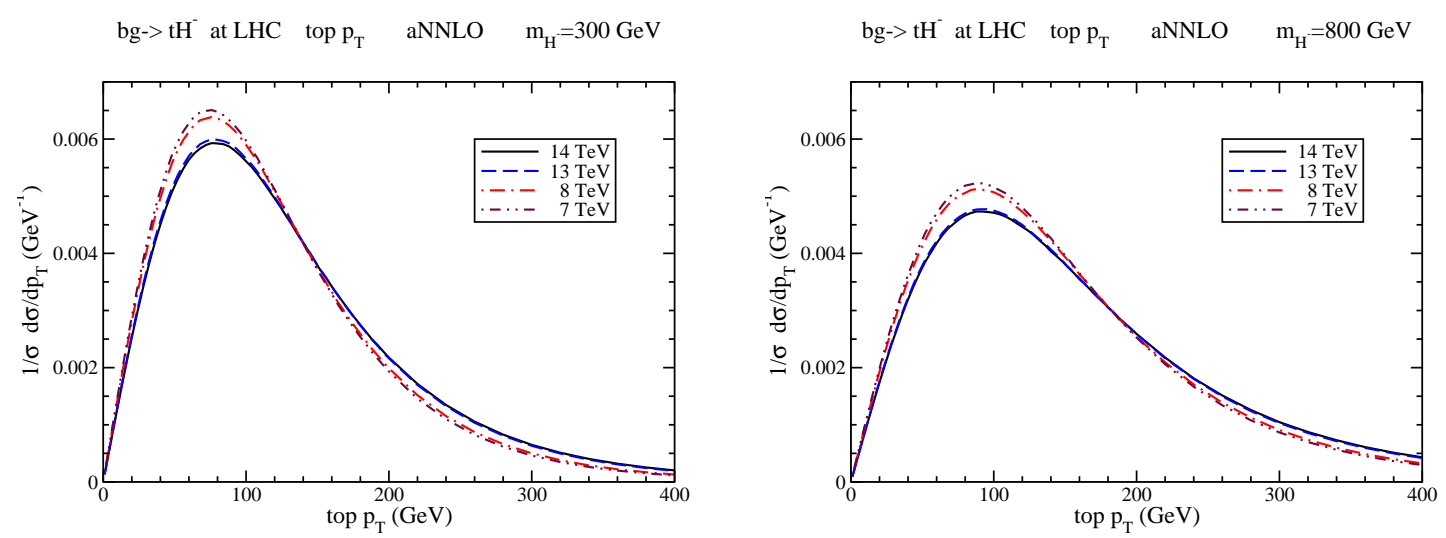

Figure 2: Normalized top-quark $p_{T}$ distributions for $t H^{-}$production with $m_{H}=300 \mathrm{GeV}$ (left) and 800 $\mathrm{GeV}$ (right). 
We continue with the transverse momentum distributions of the top quark in $t H^{-}$production. In Fig. 2 we show the aNNLO normalized top-quark $p_{T}$ distributions, $(1 / \sigma) d \sigma / d p_{T}$. The left plot shows results for a charged Higgs mass of $300 \mathrm{GeV}$ while the plot on the right is for $800 \mathrm{GeV}$. We find significant corrections at all LHC energies. The shapes of the normalized distributions change as the energy is increased: at lower LHC energies the peak is higher and it appears at lower $p_{T}$, while at larger $p_{T}$ the curves from higher energies are larger.
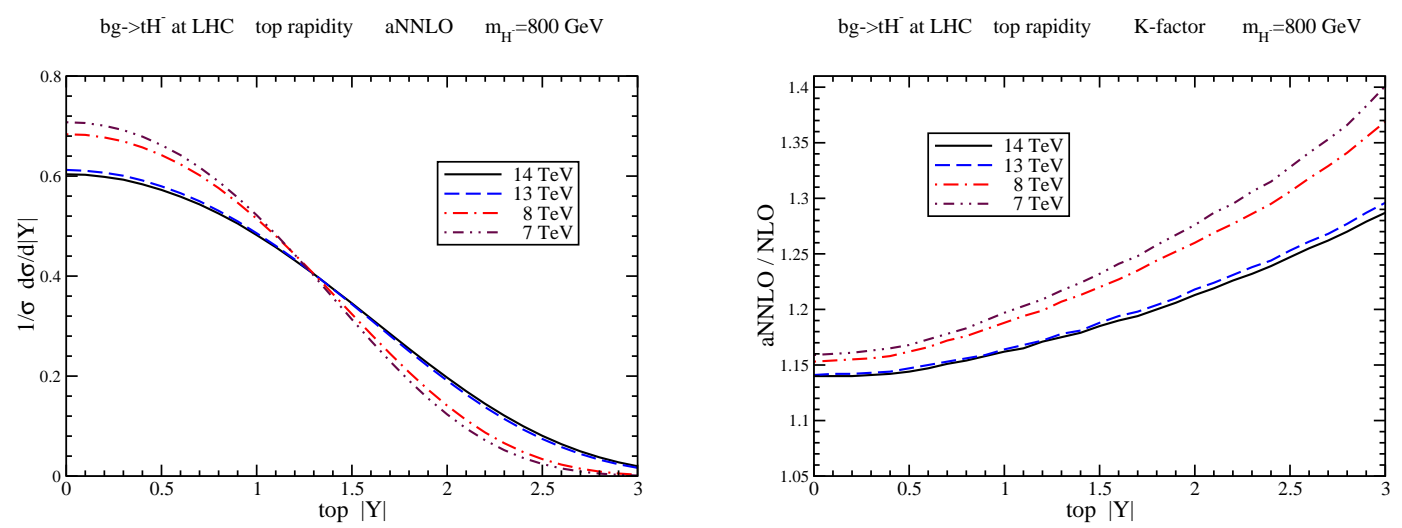

Figure 3: Normalized top-quark rapidity distributions (left), and $K$ factors (right) for $t H^{-}$production with $m_{H}=800 \mathrm{GeV}$.

In Fig. 3 we show results for the top-quark rapidity distributions in $t H^{-}$production with a charged Higgs mass of $800 \mathrm{GeV}$. The left plot shows the aNNLO normalized top-quark rapidity distributions, $(1 / \sigma) d \sigma / d|Y|$. Again, the shape of the normalized distributions changes with energy, with the curves at higher LHC energies being higher at larger rapidities. The plot on the right shows the aNNLO/NLO $K$ factors. We find significant corrections, especially at large rapidities. The $K$ factors are larger at lower LHC energies, as expected.

\section{3. $H^{-} W^{+}$production}

We continue with the associated production of a charged Higgs boson with a $W$ boson, via the process [9]

$$
b\left(p_{1}\right)+\bar{b}\left(p_{2}\right) \rightarrow H^{-}\left(p_{3}\right)+W^{+}\left(p_{4}\right) .
$$

We define $s=\left(p_{1}+p_{2}\right)^{2}, t=\left(p_{1}-p_{3}\right)^{2}, u=\left(p_{2}-p_{3}\right)^{2}$, and $s_{4}=s+t+u-m_{H}^{2}-m_{W}^{2}$. At partonic threshold $s_{4} \rightarrow 0$. Soft-gluon corrections are important for this process [10]. In addition to the softgluon terms, here we also calculate leading terms of purely collinear origin, $\ln ^{k}\left(s_{4} / m_{H}^{2}\right)$ [10].

The NNLO collinear and soft-gluon corrections are [10]

$$
\frac{d^{2} \hat{\sigma}_{\mathrm{aNNLO}}^{(2) b \bar{b} \rightarrow H^{-} W^{+}}}{d t d u}=F_{L O}^{b \bar{b} \rightarrow H^{-} W^{+}} \frac{\alpha_{s}^{2}}{\pi^{2}}\left\{-C_{3}^{(2)} \frac{1}{m_{H}^{2}} \ln ^{3}\left(\frac{s_{4}}{m_{H}^{2}}\right)+\sum_{k=0}^{3} C_{k}^{(2)}\left[\frac{\ln ^{k}\left(s_{4} / m_{H}^{2}\right)}{s_{4}}\right]_{+}\right\} \text {. }
$$

In Fig. 4 we show the aNNLO charged Higgs $p_{T}$ and rapidity distributions in $\mathrm{H}^{-} \mathrm{W}^{+}$production with $\tan \beta=1$, using MMHT2014 NNLO pdf [8]. We find significant corrections for both 

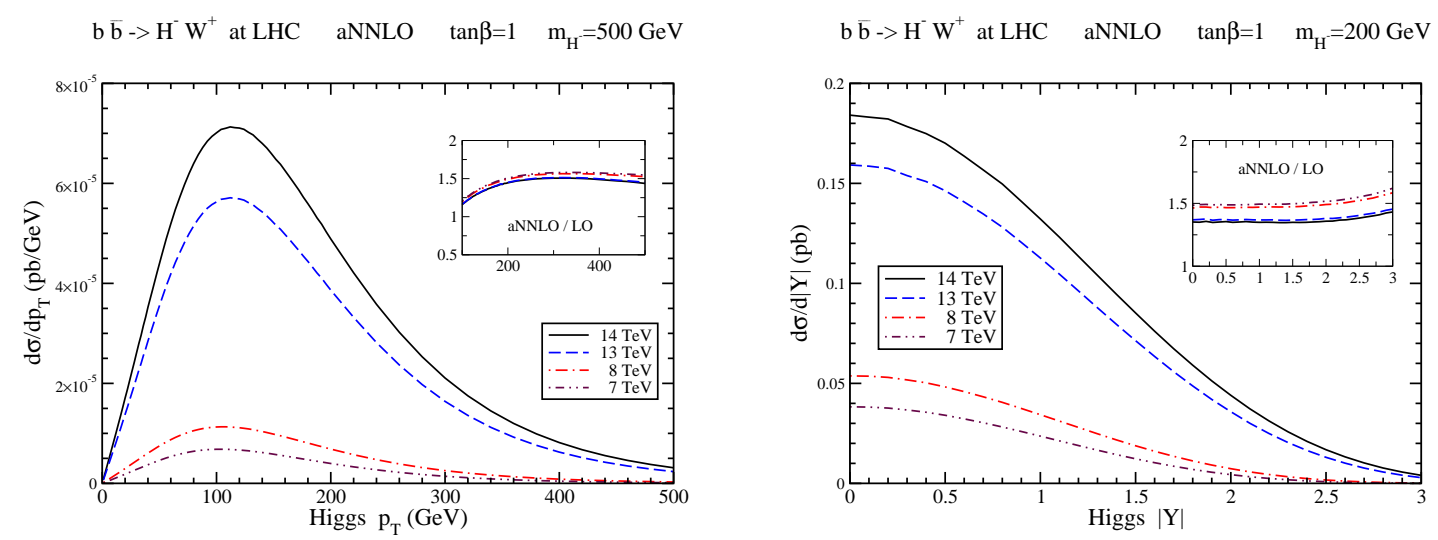

Figure 4: Charged Higgs $p_{T}$ distributions with $m_{H}=500 \mathrm{GeV}$ (left), and rapidity distributions with $m_{H}=$ $200 \mathrm{GeV}$ (right), for $H^{-} W^{+}$production with $\tan \beta=1$.

distributions, as shown in the inset plots. We note that the results using the CT14 NNLO pdf [11] are very similar.

\section{References}

[1] A. Belyaev et al., Phys. Rev. D 65, 031701 (2002); JHEP 06 (2002) 059; S.-H. Zhu, Phys. Rev. D 67, 075006 (2003); G.-P. Gao et al., Phys. Rev. D 66, 015007 (2002); T. Plehn, Phys. Rev. D 67, 014018 (2003); E.L. Berger et al., Phys. Rev. D 71, 115012 (2005); W. Peng et al., Phys. Rev. D 73, 015012 (2006); S. Dittmaier et al., Phys. Rev. D 83, 055005 (2011); M. Flechl et al., Phys. Rev. D 91, 075015 (2015); C. Degrande et al., JHEP 10 (2015) 145.

[2] N. Kidonakis, JHEP 05 (2005) 011 [hep-ph/0412422].

[3] N. Kidonakis, Phys. Rev. D 82, 054018 (2010) [arXiv:1005.4451 [hep-ph]].

[4] N. Kidonakis, Phys. Rev. D 94, 014010 (2016) [arXiv:1605.00622 [hep-ph]].

[5] N. Kidonakis, Phys. Rev. D 90, 014006 (2014) [arXiv:1405.7046 [hep-ph]]; Phys. Rev. D 91, 031501 (2015) [arXiv:1411.2633 [hep-ph]]; Phys. Rev. D 91, 071502 (2015) [arXiv:1501.01581 [hep-ph]]; Phys. Rev. D 96, 034014 (2017) [arXiv:1612.06426 [hep-ph]].

[6] N. Kidonakis, Phys. Rev. Lett. 102, 232003 (2009) [arXiv:0903.2561 [hep-ph]].

[7] N. Kidonakis, Int. J. Mod. Phys. A 19, 1793 (2004) [hep-ph/0303186]; Mod. Phys. Lett. A 19, 405 (2004) [hep-ph/0401147].

[8] L.A. Harland-Lang, A.D. Martin, P. Molytinski, and R.S. Thorne, Eur. Phys. J. C 75, 204 (2015) [arXiv:1412.3989 [hep-ph]].

[9] D.A. Dicus, J.L. Hewett, C. Kao, and T.G. Rizzo, Phys. Rev. D 40, 787 (1989).

[10] N. Kidonakis, arXiv:1704.08549 [hep-ph].

[11] S. Dulat, T.-J. Hou, J. Gao, M. Guzzi, J. Huston, P. Nadolsky, J. Pumplin, C. Schmidt, D. Stump, and C.-P. Yuan, Phys. Rev. D 93, 033006 (2016) [arXiv:1506.07443 [hep-ph]]. 